\title{
Cellular Basis of Bitter-Driven Aversive Behaviors in Drosophila Larva
}

\author{
Jaekyun Choi, ${ }^{1,}$, Seungyun Yu, ${ }^{1,}$, Min Sung Choi, ${ }^{1}$ Sooin Jang, ${ }^{1}$ I Joon Han, ${ }^{2}$ G. Larisa Maier, ${ }^{3}$ \\ Simon G. Sprecher, ${ }^{3}$ and (D) Jae Young Kwon ${ }^{1}$
}

https://doi.org/10.1523/ENEURO.0510-19.2020

${ }^{1}$ Department of Biological Sciences, Sungkyunkwan University, Suwon 16419, Republic of Korea, ${ }^{2}$ School of Medicine, Sungkyunkwan University, Suwon 16419, Republic of Korea, and ${ }^{3}$ Department of Biology, Institute of Zoology, University of Fribourg, Fribourg $\mathrm{CH}-1700$, Switzerland

\begin{abstract}
Feeding, a critical behavior for survival, consists of a complex series of behavioral steps. In Drosophila larvae, the initial steps of feeding are food choice, during which the quality of a potential food source is judged, and ingestion, during which the selected food source is ingested into the digestive tract. It remains unclear whether these steps employ different mechanisms of neural perception. Here, we provide insight into the two initial steps of feeding in Drosophila larva. We find that substrate choice and ingestion are determined by independent circuits at the cellular level. First, we took 22 candidate bitter compounds and examined their influence on choice preference and ingestion behavior. Interestingly, certain bitter tastants caused different responses in choice and ingestion, suggesting distinct mechanisms of perception. We further provide evidence that certain gustatory receptor neurons (GRNs) in the external terminal organ (TO) are involved in determining choice preference, and a pair of larval pharyngeal GRNs is involved in mediating both avoidance and suppression of ingestion. Our results show that feeding behavior is coordinated by a multistep regulatory process employing relatively independent neural elements. These findings are consistent with a model in which distinct sensory pathways act as modulatory circuits controlling distinct subprograms during feeding.
\end{abstract}

Key words: Drosophila; neural circuit; taste

\section{Significance Statement}

Here, we provide molecular and cellular evidence that feeding can indeed be dissected into two distinct steps, namely the determination of preference and initiation of ingestion. We find that bitter tastants have individual characteristics when negatively affecting feeding, with most chemicals negatively affecting both preference and ingestion, and certain chemicals negatively affecting only preference. These characteristics are due to different sensory neurons being responsible for detecting different bitter compounds. The different sensory neurons appear to act in relatively independent neural circuits to differentially affect the multiple steps that comprise feeding.

\section{Introduction}

The regulation of feeding is critical for the survival of animals. Feeding is comprised of a series of behavioral modules or subprograms that can be regulated at multiple levels (Pool and Scott, 2014). At the initiation step of a meal, the chemical composition of a food source provides

\footnotetext{
Received December 8, 2019; accepted March 16, 2020; First published March 20, 2020.

The authors declare no competing financial interests.
}

important information regarding the palatability and quality of food, which in turn is essential for the decision of whether to feed on the respective food. Sweet taste is generally recognized as a preferred source of nutrients, while bitter taste is generally recognized as toxic material to be avoided. Animals can regulate feeding by sensing

Author contributions: S.G.S. and J.Y.K. designed research; J.C. and S.Y. performed research; J.C., S.Y., M.S.C., S.J., I.J.H., G.L.M., S.G.S., and J.Y.K. analyzed data; S.Y., M.S.C., I.J.H., G.L.M., S.G.S., and J.Y.K. wrote the paper. 
bitterness, subsequently avoiding the bitter food, and suppressing ingestion. Drosophila larvae have been used as an effective model to study the cellular and molecular bases of behavioral responses caused by bitterness. (ElKeredy et al., 2012; Apostolopoulou et al., 2014; König et al., 2014; Choi et al., 2016; Kim et al., 2016; van Giesen et al., 2016b).

The major chemosensory organs of Drosophila larvae are located in bilaterally symmetrical pairs on the head, and are composed of three external sense organs: the terminal organ (TO), ventral organ (VO), and dorsal organ (DO) as well as three sets of pharyngeal sensilla (Singh and Singh, 1984; Stocker, 1994; Python and Stocker, 2002; Gendre et al., 2004; Gerber and Stocker, 2007). Also, numerous studies have provided functional evidence that these organs are indeed involved in taste perception (Python and Stocker, 2002; Apostolopoulou et al., 2014, 2015; Choi et al., 2016; Kim et al., 2016; van Giesen et al., 2016b).

Members of the gustatory receptor $(\mathrm{Gr})$, ionotropic receptor $(I R)$, and pickpocket (ppk) gene families are involved in chemosensory perception, and are expressed in the larval gustatory neurons (Liu et al., 2003; Colomb et al., 2007; Thorne and Amrein, 2008; Kwon et al., 2011; Mishra et al., 2013; Stewart et al., 2015). The expression of some Ir, Ppk, and Gr genes have been mapped to neurons of individual sensilla in the TO (Rist and Thum, 2017), and expression of Gr-GAL4 drivers has been mapped to individual GRNs in the TO and pharyngeal sense organs (Kwon et al., 2011; Choi et al., 2016). The Gr-to-neuron map of the TO and pharyngeal sense organs that was constructed based on Gr-GAL4 driver expression shows that many GRNs in Drosophila larva express receptors that have been associated with bitter taste in the adult fly, suggesting the sensing of bitterness by these GRNs. However, co-expression of several receptors differ in the larva and the adult, and numerous Grs are stage specific. Accumulating evidence for the multimodality of taste sensing in the larva (van Giesen et al., 2016b) and complex receptor co-expression requirements for taste discrimination in the fly raise the question of how these neurons coordinate taste perception (Jaeger et al., 2018).

We here show that feeding initiation in Drosophila larvae can be divided into two distinct steps, choice and ingestion, with choice being the process of selecting a potential

This work was supported by the National Research Foundation of Korea (NRF) Grant NRF-2016R1D1A1B03932743 and 2019R1F1A1059833 (to J.Y.K.); the Ministry of Science, ICT and Future Planning Grant NRF2017R1A2B4007280 (to M.S.C.); and by the Schweizerischer Nationalfonds zur Förderung der wissenschaftlichen Forschung (SNF) Grant 31003A_149499 (to S.G.S.)

a J.C. and S.Y. contributed equally to this work.

Acknowledgements: We thank the Bloomington Drosophila Stock Center for providing flies.

Correspondence should be addressed to Simon G. Sprecher at simon. sprecher@gmail.com or Jae Young Kwon at jykwon@skku.edu.

https://doi.org/10.1523/ENEURO.0510-19.2020 Copyright (C) 2020 Choi et al.

This is an open-access article distributed under the terms of the Creative Commons Attribution 4.0 International license, which permits unrestricted use, distribution and reproduction in any medium provided that the original work is properly attributed. food source, and ingestion the process of moving food down the pharyngeal tract into the digestive system. We tested a wide panel of twenty-two putative bitter compounds and found that some tastants have different impacts on ingestion and choice, contrary to our initial assumption that tastants would generally cause similar responses in these two behavioral paradigms. Using molecular genetics and calcium imaging, we provide evidence that caffeine (CAF) is detected by a pair of neurons in the dorsal pharyngeal sensilla (DPS) to induce avoidance movement and reduction of ingestion, and denatonium is detected by neurons in the TO to induce only avoidance movement.

\section{Materials and Methods}

\section{Drosophila stocks and transgenic flies}

Flies were cultured on standard cornmeal agar medium at room temperature $\left(23 \pm 2^{\circ} \mathrm{C}\right)$. $w C S$ was used a control for behavioral assays. All Gr-GAL4 transgenic lines used in this study were previously described (Kwon et al., 2011). The following fly lines were used: $\mathrm{Gr}_{3} 3 a^{1}$ (Moon et al., 2009), UAS-Kir2.1 (Baines et al., 2001), C7-GAL4 (van Giesen et al., 2016b), UAS-Gr33a (Moon et al., 2009), UAS-Gr59c (Weiss et al., 2011), and UAS-GCaMP6m (Chen et al., 2013).

\section{Tastants}

The highest purity tastants commercially available were purchased for use in behavioral assays. Stock solutions of the tastants dissolved in water were mixed with 1\% agarose solution (ingestion assay) or 1.5\% agarose solution (choice assay) to prepare the plates used in the behavioral assays. The following 22 bitter compounds were selected to test in this study: atropine (ATR; A0132, Sigma), berberine chloride (BER; B3251, Sigma), CAF (27600, Sigma), (+)-catechin (CAT; ALX385-017, Enzo), coumarin (COU; C4261, Sigma), N,Ndiethyl-m-toluamide (DEET; PS-902, Supelco), denatonium benzoate (DEN; D5765, Aldrich), escin (ESC; E1378, Sigma), gallic acid (GAA; G7384, Sigma), gibberellic acid (GIA; 63492, Aldrich), harmaline (HAR; 51330, Aldrich), (-)-lobeline hydrochloride (LOB; 141879, Aldrich), (-)-nicotine (NIC; 36733, Fluka), N-phenylthiourea (PTU; P7629, Sigma), quinine hydrochloride dihydrate (QUI; 22630, Sigma), saponin (SAP; 102855, MP Bio), D-(+)-sucrose octaacetate (SOA; 84112, Fluka), strychnine nitrate (STR; S0093, TCI), tannic acid (TAA; 194859, MP Bio), theobromine (THB; T4500, Sigma), theophylline anhydrous (TPH; 103024, MP Bio), and umbelliferone (UMB; 93979 , Sigma).

\section{Behavioral assays}

Third instar larvae were used for all behavioral experiments. All assays were conducted with 5 D-old larvae after egg laying. All larvae were thoroughly washed with distilled water. For the ingestion assay, we followed a previously described protocol (Choi et al., 2016). Briefly, 60$\mathrm{mm}$ Petri dishes (SPL 10060) were filled with 1\% agarose solution $+1 \%$ indigo carmine (Sigma, 57000; control 
plates), or $1 \%$ agarose solution $+1 \%$ indigo carmine + bitter substance (experimental plates). Thirty third instar larvae were placed in the center of the plate. After $90 \mathrm{~min}$ of feeding, larvae were collected and washed with distilled water. Next, larvae were homogenized using a pistil in $1 \mathrm{M}$ $60-\mu \mathrm{l}$ L-ascorbic acid (Sigma, A7506). After centrifugation at $13,200 \mathrm{rpm}$ for $10 \mathrm{~min}$, the blue supernatant was transferred to a 1- $\mu \mathrm{m}$-pore FAPD column and centrifuged at $13,200 \mathrm{rpm}$ for $3 \mathrm{~min}$. The filtered supernatant was transferred to a 96-well plate (SPL 30096) and absorbance was measured at $630 \mathrm{~nm}$ with a spectrophotometer (BioTek EL800). The relative ingestion index (I.I.) was derived by calculating the difference in absorbance O.D. (optical density) between the control and experimental groups: I.I. = (experimental O.D. - empty O.D.) - (dye-only control O.D. - empty O.D.)/(dye-only control O.D. - empty O.D.). The dye only control O.D. was measured for every experiment, for every genotype, on the same day. An I.I. value of 0 indicates that larvae on the test plate ate as much as larvae on the dye-only control plate, while I.I. $=-1$ indicates that larvae on the test plate did not eat at all, and I.I. $>0$ indicates that larvae on the test plate ate more than larvae on the dye-only control plate.

For the choice preference assay, we used a previously described method with modifications (Kim et al., 2016). Ninety-millimeter Petri dishes (SPL 10096) were first filled with $1.5 \%$ agarose solution and allowed to cool. After dividing the cooled agarose into quadrants and carefully removing the second and fourth agarose quadrants, the now empty quadrants were filled with $1.5 \%$ agarose containing a bitter substance. After the plate fully solidified, 20 third instar larvae were placed in the center of the prepared quadrant plate. The number of larvae on each quadrant were counted after $8 \mathrm{~min}$, with the first and third quadrant being the agarose only control and the second and fourth quadrant containing the bitter substance. The choice preference index (P.I.) was calculated as follows: P.I. $=\left[\mathrm{N}_{\text {bitter tastant }}-\mathrm{N}_{\text {agarose only }}\right] /\left[\mathrm{N}_{\text {total }}\right]$. A negative P.I. value indicates that the larvae showed aversive behavior toward the tested putative bitter substance.

For mouth-hook contraction, body wall contraction, and bending behavior assay, we used a previously described method with some modifications (Shen, 2012; Bhatt and Neckameyer, 2013). 90-mm Petri dishes (SPL 10096) were filled with $1 \%$ agarose (control plate), or $1 \%$ agarose with bitter substance (experimental plate). One larva was placed on the center of the plate, and video recording was done for $60 \mathrm{~s}$ after a 90 -s acclimation. We analyzed the video files by counting the number of each mouth-hook contraction, body wall contraction, and bending behavior. Mouth-hook contraction was assessed as pharyngeal muscle movement, body wall contraction is locomotion movement, and bending was assessed as when the larval body was bent at an angle $>45^{\circ}$. Each behavior was compared between larvae on $1 \%$ agarose plate (control), or $1 \%$ agarose plate with bitter substance (experiment).

\section{GCaMP imaging}

To record calcium responses, early stage third instar larvae were dissected in modified AHL, adult hemolymph- like-saline (108 mm NaCl, $5 \mathrm{~mm} \mathrm{KCl,} 8.2 \mathrm{~mm} \mathrm{MgCl}$, $4 \mathrm{~mm}$ $\mathrm{NaHCO}_{3}, 1 \mathrm{~mm} \mathrm{NaH} \mathrm{PO}_{4}$, and $5 \mathrm{~mm}$ HEPES, pH 7.5 in Millipore water). To enable recording in DP1 and DP2, the cuticle surrounding the tip of the head was removed and the head was introduced into the microfluidic chip chamber to expose chemosensory organs to the liquid passing through the channel (van Giesen et al., 2016a). A drop of $2 \%$ agarose diluted in $\mathrm{AHL}$ saline was used to close the channel. Measurements were conducted as follows: a 10$s$ period of washing (Millipore water) followed by a 20-s period of stimulation and another $10 \mathrm{~s}$ of washing. Changes in fluorescence were calculated as follows:

$$
\Delta \mathrm{F} / \mathrm{F}(\%)=\left(\mathrm{F}_{\text {peak }}-\mathrm{F}_{0}\right) * 100 / \mathrm{F}_{0} .
$$

$F_{0}$ was calculated from five frames during the prestimulation phase of the first 100 -frame time period. $F_{\text {peak }}$ was taken as the point of highest intensity measured during the time of stimulation. A Zeiss LSM 700 confocal microscope was used for imaging. For the analysis of calcium imaging measurements, ImageJ was used and changes in fluorescence were calculated in Microsoft Excel.

\section{Experimental design and statistical analyses}

Statistical analyses were conducted using GraphPad Prism 5 and IBM SPSS Statistics 20. Behavior data are mostly presented as a box plot, with the middle line representing the median, + the mean, and the box boundaries and whiskers representing $25 \% / 75 \%$ and $10 \% / 90 \%$, respectively. For Figures 2, $3 A, 4 A, 5 A, 6 A$, normality was tested. Data were considered to be normally distributed if it passed either the Kolmogorov-Smirnov test or the Shapiro-Wilk normality test. For Figure 2, one-way ANOVA followed by Dunnett's multiple comparison test was used. For Figures $3 A, 4 A, 6 A$, one-way ANOVA followed by the Newman-Keuls method was performed if the data were normally distributed. If the data were not normally distributed, the Kruskal-Wallis test followed by Dunn's multiple comparison test was performed. For Figure $5 A$, one-way ANOVA was followed by uncorrected Fisher's least significant difference (LSD) test. For Figures 3B,C, 4B,C, 5B,C, $6 B$, two-way ANOVA followed by the Bonferroni post hoc test was performed. The Mann-Whitney $U$ test was used for pair-wise comparison of GCaMP imaging data. Asterisks shown in figures signify statistical significance $(* p<0.05, * * p<0.01, * * * p<0.001)$. Additional details are described in the figure legends and Table 1.

\section{Results}

\section{Individual putative bitter tastants elicit distinct ingestion and choice preference behavior responses}

In two previous studies, we measured larval preference (Kim et al., 2016) and ingestion behavior (Choi et al., 2016) toward putative bitter chemicals that were expected to elicit an aversive response. Interestingly, certain chemicals elicited differential responses between the two paradigms. As an example, denatonium caused larval avoidance in a dose-dependent manner (Kim et al., 2016), but did not affect ingestion even at the highest 
Table 1: Statistical analysis summary

\begin{tabular}{|c|c|c|c|c|c|c|c|}
\hline & Figure & Test comparison & Statistical test & Result & Post hoc test & post-hoc comparison & $\begin{array}{c}\text { Adjusted } \\
\text { P value }\end{array}$ \\
\hline \multirow[t]{9}{*}{$2 A$} & Mouth-hook & Comparisons be- & One-way ANOVA & $F_{(3,102)}=7.901$, & Dunnett's multiple & Control vs CAF & 0.001 \\
\hline & contraction & tween tastants & & $P<0.0001$ & comparison test & Control vs DEN & $>0.999$ \\
\hline & & & & & & Control vs NIC & $<0.001$ \\
\hline & Bending & & & $F_{(3,102)}=8.846$ & & Control vs CAF & 0.092 \\
\hline & & & & $P<0.0001$ & & Control vs DEN & 0.026 \\
\hline & & & & & & Control vs NIC & 0.026 \\
\hline & Body wall & & & $F_{(3,102)}=16.94$ & & Control vs CAF & 0.598 \\
\hline & contraction & & & $P<0.0001$ & & Control vs DEN & 0.628 \\
\hline & & & & & & Control vs NIC & $<0.001$ \\
\hline \multirow[t]{9}{*}{$2 B$} & Mouth-hook & Comparisons be- & One-way ANOVA & $F_{(3,76)}=4.355$ & Dunnett's multiple & Control vs CAF & 0.473 \\
\hline & contraction & tween tastants & & $P=0.0069$ & comparison test & Control vs DEN & 0.060 \\
\hline & & & & & & Control vs NIC & 0.995 \\
\hline & Bending & & & $F_{(3,76)}=4.773$ & & Control vs CAF & 0.312 \\
\hline & & & & $P=0.0042$ & & Control vs DEN & 0.923 \\
\hline & & & & & & Control vs NIC & 0.078 \\
\hline & Body wall & & & $F_{(3,76)}=11.90$ & & Control vs CAF & $>0.999$ \\
\hline & contraction & & & $P<0.0001$ & & Control vs DEN & 0.765 \\
\hline & & & & & & Control vs NIC & $<0.001$ \\
\hline \multirow[t]{6}{*}{$2 C$} & Mouth-hook & Comparisons be- & One-way ANOVA & $F_{(2,56)}=0.2104$, & Dunnett's multiple & Control vs SUC & $>0.999$ \\
\hline & contraction & tween tastants & & $P=0.8109$ & comparison test & Control vs FRU & 0.793 \\
\hline & Bending & & Kruskal-Wallis test & $0.9784, P=$ & Dunn's multiple & Control vs SUC & $>0.05$ \\
\hline & & & & 0.6131 & comparison test & Control vs FRU & $>0.05$ \\
\hline & Body wall & & One-way ANOVA & $F_{(2,56)}=0.1099$, & Dunnett's multiple & Control vs SUC & 0.990 \\
\hline & contraction & & & $P=0.8961$ & comparison test & Control vs FRU & 0.863 \\
\hline \multirow[t]{10}{*}{$3 A$} & & Comparisons be- & Kruskal-Wallis test & $39.44, P<$ & Dunn's multiple & UAS-Kir2.1/+ vs Gr33a >Kir2.1 & $<0.05$ \\
\hline & & tween genotypes & & 0.0001 & comparison test & Gr33a-GAL4/+ vs Gr33a $>$ Kir2.1 & $<0.05$ \\
\hline & & & & & & UAS-Kir2.1/+ vs Gr39b > Kir2.1 & $<0.05$ \\
\hline & & & & & & Gr39b-GAL4/+ vs Gr39b >Kir2.1 & $<0.01$ \\
\hline & & & & & & UAS-Kir2.1/+ vs Gr23a $>$ Kir2.1 & $>0.05$ \\
\hline & & & & & & Gr23a-GAL4/+ vs Gr23a $>$ Kir2.1 & $>0.05$ \\
\hline & & & & & & UAS-Kir2.1/+ vs Gr22a >Kir2.1 & $>0.05$ \\
\hline & & & & & & Gr22a-GAL4/+ vs Gr22a $>$ Kir2.1 & $>0.05$ \\
\hline & & & & & & UAS-Kir2.1/+ vs Gr36b > Kir2.1 & $>0.05$ \\
\hline & & & & & & Gr36b-GAL4/+ vs Gr36b $>$ Kir2.1 & $>0.05$ \\
\hline \multirow[t]{10}{*}{$3 B$} & & Interaction between & Two-way ANOVA & $F_{(9,356)}=4.149$ & Bonferroni's multiple & Control vs $100 \mathrm{mM} \mathrm{CAF} \mathrm{(Gr33a-GAL4/+)}$ & $<0.05$ \\
\hline & & genotype and & & $P<0.0001$ & comparison test & Control vs 100 mM CAF (Gr33a > Kir2.1) & $<0.05$ \\
\hline & & chemical & & & & Control vs $100 \mathrm{mM} \mathrm{CAF}(\mathrm{Gr} 39 \mathrm{~b}-\mathrm{GAL} 4 /+)$ & $<0.05$ \\
\hline & & & & & & Control vs $100 \mathrm{mM}$ CAF (Gr39b>kir2.1) & $>0.05$ \\
\hline & & & & & & Control vs $100 \mathrm{mM} \mathrm{CAF} \mathrm{(Gr23a-GAL4/+)}$ & $<0.01$ \\
\hline & & & & & & Control vs $100 \mathrm{mM}$ CAF (Gr23a>kir2.1) & $<0.01$ \\
\hline & & & & & & Control vs $100 \mathrm{mM} \mathrm{CAF}(\mathrm{Gr} 22 \mathrm{a}-\mathrm{GAL} 4 /+)$ & $>0.05$ \\
\hline & & & & & & Control vs $100 \mathrm{mM}$ CAF (Gr22a > kir2.1) & $>0.05$ \\
\hline & & & & & & Control vs $100 \mathrm{mM} \mathrm{CAF}(\mathrm{Gr} 36 b-G A L 4 /+)$ & $<0.01$ \\
\hline & & & & & & Control vs $100 \mathrm{mM} \mathrm{CAF}(G r 36 b>k i r 2.1)$ & $>0.05$ \\
\hline \multirow[t]{10}{*}{$3 C$} & & Interaction between & Two-way ANOVA & $F_{(9,356)}=1.837$ & Bonferroni's multiple & Control vs $100 \mathrm{mM} \mathrm{CAF} \mathrm{(Gr33a-GAL4/+)}$ & $>0.05$ \\
\hline & & genotype and & & $P=0.0606$ & comparison test & Control vs 100 mM CAF (Gr33a> Kir2.1) & $>0.05$ \\
\hline & & chemical & & & & Control vs $100 \mathrm{mM} \mathrm{CAF}(\mathrm{Gr} 39 \mathrm{~b}-\mathrm{GAL} 4 /+)$ & $>0.05$ \\
\hline & & & & & & Control vs $100 \mathrm{mM}$ CAF (Gr39b>kir2.1) & $>0.05$ \\
\hline & & & & & & Control vs $100 \mathrm{mM} \mathrm{CAF}(\mathrm{Gr} 23 a-G A L 4 /+)$ & $<0.01$ \\
\hline & & & & & & Control vs $100 \mathrm{mM}$ CAF (Gr23a>kir2.1) & $<0.05$ \\
\hline & & & & & & Control vs 100 mM CAF (Gr22a-GAL4/+) & $>0.05$ \\
\hline & & & & & & Control vs $100 \mathrm{mM}$ CAF (Gr22a>kir2.1) & $>0.05$ \\
\hline & & & & & & Control vs $100 \mathrm{mM} \mathrm{CAF}(\mathrm{Gr} 36 \mathrm{~b}-\mathrm{GAL} 4 /+)$ & $>0.05$ \\
\hline & & & & & & Control vs $100 \mathrm{mM}$ CAF (Gr36b>kir2.1) & $>0.05$ \\
\hline \multirow[t]{8}{*}{$4 A$} & & Comparisons be- & One-way ANOVA & $F_{(10,64)}=15.38$ & Newman-Keuls mul- & UAS-Gr33a/+ vs Gr33a $>$ Gr33a & $<0.001$ \\
\hline & & tween genotypes & & $P<0.0001$ & tiple comparison & Gr33a-GAL4/+ vs Gr33a $>$ Gr33a & $<0.001$ \\
\hline & & & & & test & UAS-Gr33a/+ vs Gr39b > Gr33a & $<0.001$ \\
\hline & & & & & & Gr39b-GAL4/+ vs Gr39b>Gr33a & $<0.001$ \\
\hline & & & & & & UAS-Gr33a/+ vs Gr22d>Gr33a & $<0.001$ \\
\hline & & & & & & Gr22d-GAL4/+ vs Gr22d > Gr33a & $<0.001$ \\
\hline & & & & & & UAS-Gr33a/+ vs Gr22a $>$ Gr33a & $>0.05$ \\
\hline & & & & (Conti & ded) & & \\
\hline
\end{tabular}


Table 1: Continued

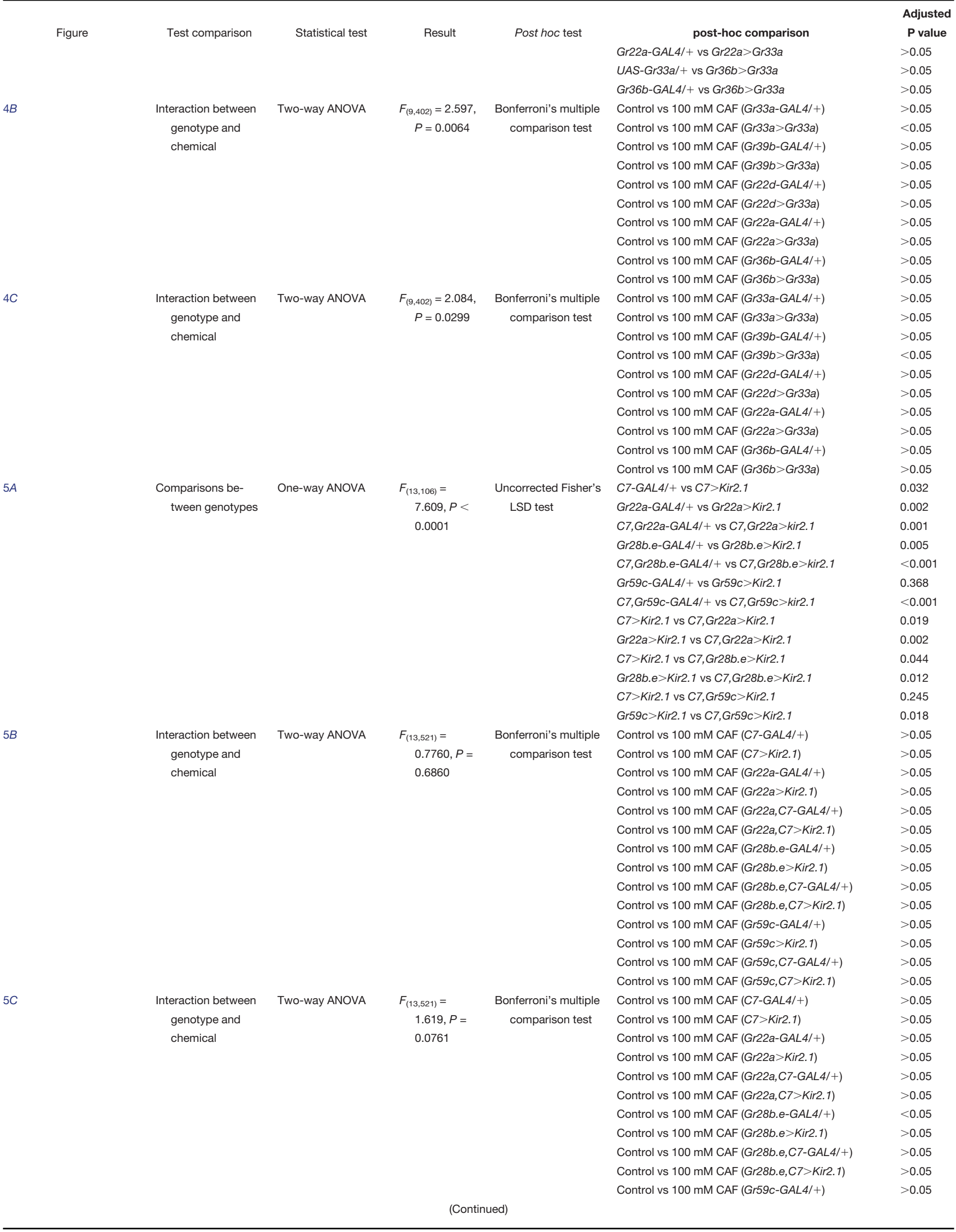


Table 1: Continued

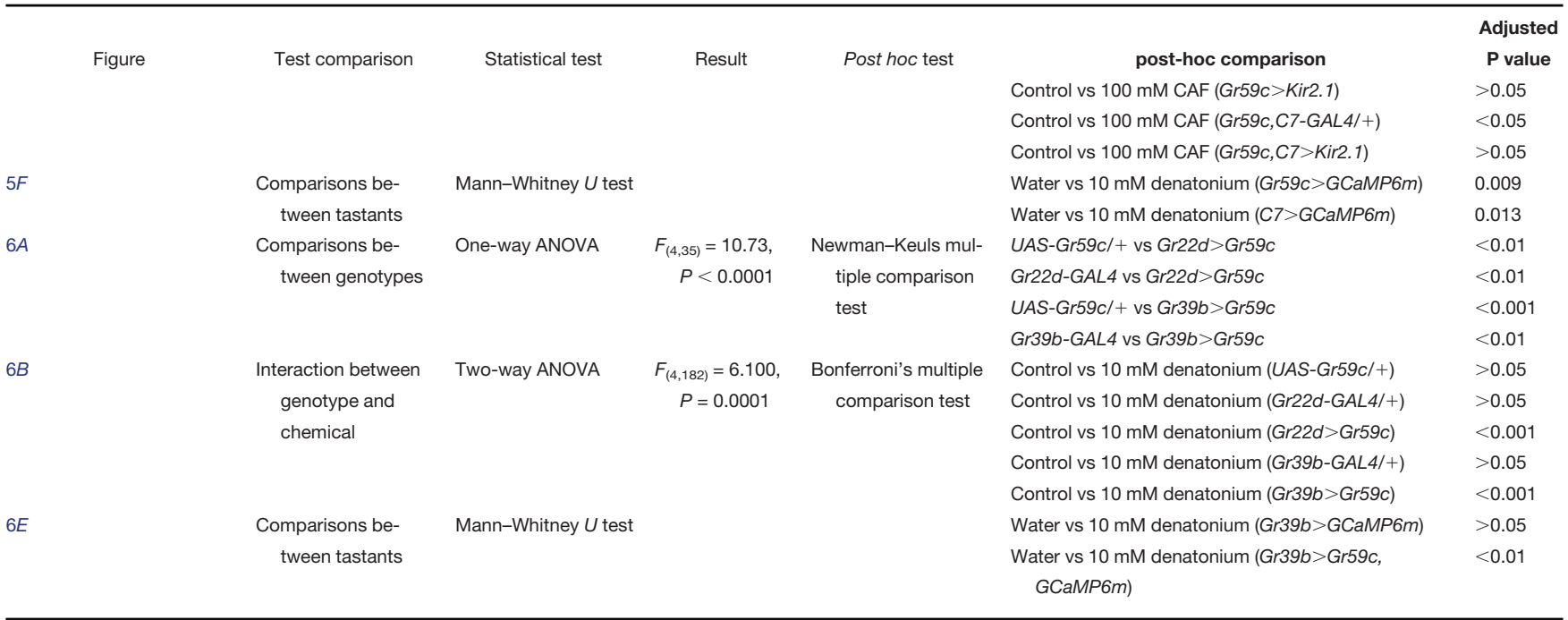

tested concentration of $10 \mathrm{~mm}$ (Choi et al., 2016). Here, we re-analyzed and quantitatively measured the two larval behaviors elicited by the bitter chemicals: ingestion (Fig. 1A) and choice behavior for substrate preference (Fig. 1B). We allowed larvae to feed for 90 min on control plates with agarose and dye or experimental plates with added tastants before quantifying and comparing the amounts ingested (Fig. 1C; for details, see Materials and Methods). An I.I. of 0 indicates that experimental larvae ingested the same amount as control larvae fed on dye only-agarose, and an I.I. of -1 indicates that experimental larvae did not ingest at all. An I.I. larger than 0 indicates that the tastant is a positive effector of ingestion, while an I.I. between 0 and -1 indicates that the tastant is a negative effector. In choice preference behavior assays, larvae were placed in the center of plates containing two quadrants with plain agarose and two quadrants with agarose and bitter tastants (Fig. 1B). We excluded the possible influence of sugar (for details, see Materials and Methods), in contrast to the previous study where sugar only and sugar plus putative bitter chemical were compared (Kim et al., 2016). The number of larvae on each quadrant was counted after $8 \mathrm{~min}$ to calculate the choice P.I. P.I. values between 0 and 1 indicate attraction, while values between 0 and -1 indicate aversion.

We found that CAF, STR, and TPH act as conventional bitter tastants that reduce ingestion and induce aversion (Fig. 1C); 100 mm CAF caused an I.I. of -0.911 , with larvae barely eating agarose containing $100 \mathrm{~mm}$ CAF, and a P.I. of -0.948 , with most larvae showing aversion toward CAF. Tastants such as DEET, COU, LOB, QUI, UMB, and BER show a similar tendency. Consistent with our previous observations, some tastants elicited differential effects on ingestion and preference behavior, which is counterintuitive to the general assumption that tastants would generally have similar effects on ingestion and preference. NIC and ESC cause a strong negative ingestion response but appear neutral or attractive in the choice preference assay (Fig. 1C). In contrast, DEN and HAR do not affect ingestion, with an I.I. close to 0 even at the highest tested concentrations (10 mm DEN and $5 \mathrm{~mm} H A R$ ), but cause a strong aversive choice preference response (Fig. 1C). These results indicate that some putative bitter tastants act independently on ingestion and choice preference, two contact chemosensation-driven behavioral paradigms that are commonly viewed as being closely related.

\section{CAF affects both mouth-hook contraction and bending, while denatonium only affects bending behavior}

In measuring taste-driven larval choice preference behavior, the determination of whether a certain chemical is an attractant or a repellent is based on where the larvae are located, i.e., on the control or experimental sectors, after a certain amount of time. We discovered that this method was a source of error in the case of measuring preference behavior to nicotine. While monitoring larvae over the entire $8 \mathrm{~min}$ of measurement after the larvae were placed in the center of the plate, we found that most of the larvae stopped movement before the $8 \mathrm{~min}$ passed (Extended Data Fig. 1-1). This indicates that larvae are not staying in the nicotine quadrants because they prefer nicotine itself, but because of possible toxic effects of nicotine that block movement. This case of nicotine is an example of misreading actual behavior due to limitations in methodology. After observing this, we decided that additional behavioral assays would be necessary for the accurate analysis of taste-driven larval behavior.

Previous studies indicated that the frequency of mouthhook contractions directly correlates to the amounts ingested (Joshi and Mueller, 1988; Wu et al., 2003, 2005). Also, lateral bending is one of the most commonly observed components of turning behavior, which is evoked when larvae decide to avoid noxious stimuli or explore new territory (Okusawa et al., 2014; Hernandez-Nunez et al., 2015). We were able to correlate these behaviors with larval responses to bitter chemicals. We counted the 
A

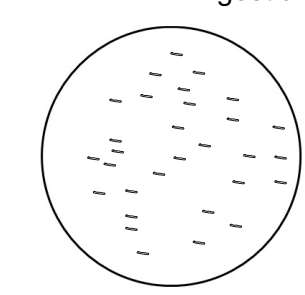

dye only
B

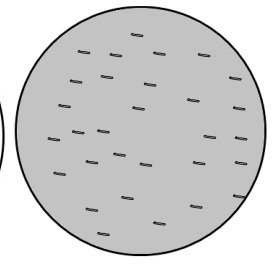

dye + tastant

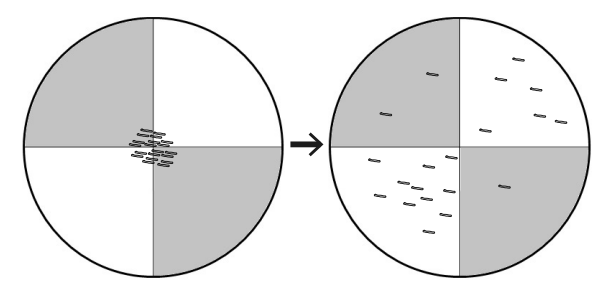

pure agarose

agarose + tastant

C

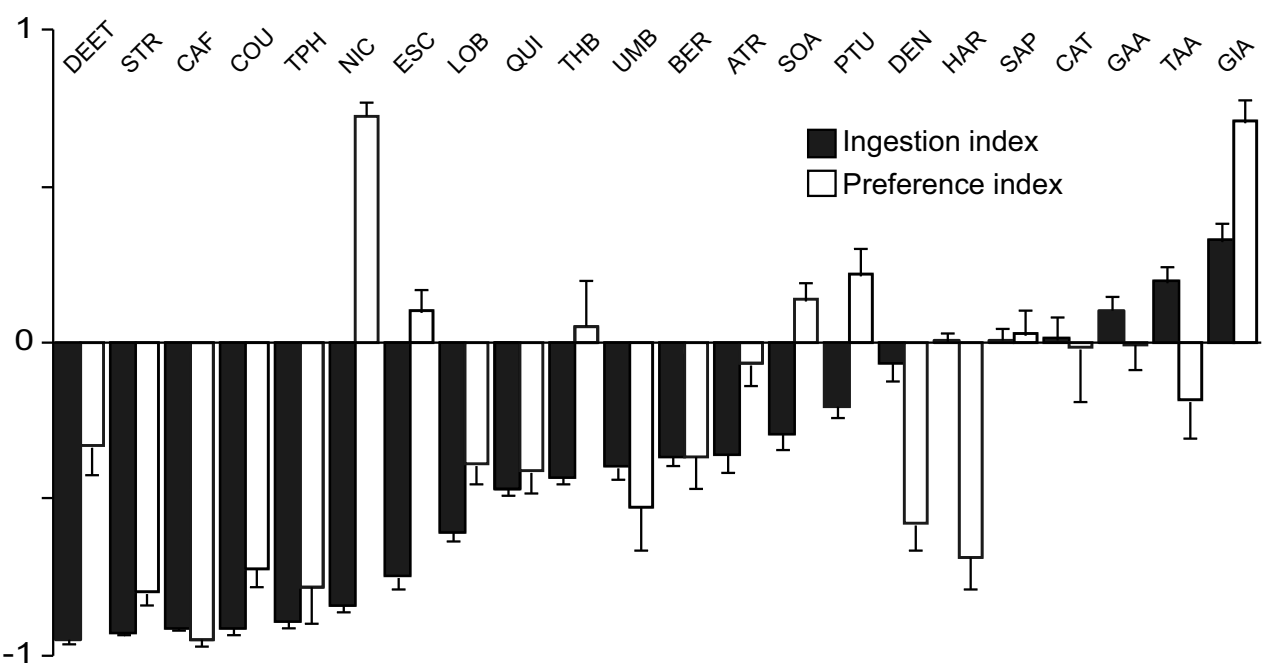

Figure 1. Responses toward putative bitter tastants in larval ingestion and choice assays. $\boldsymbol{A}$, Schematic drawing of the ingestion assay. Two agarose gel plates were used, with one containing only $1 \%$ indigo carmine dye (left, white) and one also containing a bitter substance (right, shaded). Thirty larvae were placed on each plate and allowed to forage and feed freely for 90 min. The ratio of dye ingested by the two groups was measured to calculate the I.I.. B. For the choice preference assay, larvae were placed onto a quadrant plate made with two types of agarose gel: agarose only (white quadrants) and tastant included (shaded quadrants). After $8 \mathrm{~min}$, the number of larvae on each quadrant was counted to analyze the larval behavioral response to the compound. The short lines in $\boldsymbol{A}, \boldsymbol{B}$ indicate larvae. $\boldsymbol{C}$, Comparison of ingestion and choice preference behavioral responses for the twenty-two bitter compounds tested. Compounds are arranged in order of increasing I.I. values from left to right. Each data point was derived from $6<n$. Error bars are SEM. The following concentrations were used: $10 \mathrm{~mm}$ ATR, 5 mm BER, 100 mм CAF, 10 mm CAT, 10 mm COU, $1 \%$ DEET, 10 mм DEN, 1 mм ESC, 50 mм GAA, 10 mм GIA, 5 mм HAR, 10 mм LOB, 10 mм NIC, 1 mм PTU, 10 mм QUI, $0.1 \%$ SAP, 1 mм SOA, 100 mм STR, 1 mм TAA, 30 mм THB, 100 mм TPH, and 10 mм UMB (Extended Data Figure 1-1).

frequencies of mouth-hook contractions, bending, and body wall contractions on wCS larvae exposure to CAF, denatonium, or nicotine (Fig. 2A). Mouth-hook contractions were observed to decrease on exposure to CAF compared with the agarose-only control (Fig. 2A, left), consistent with the decrease in ingestion (Fig. 1). An increase in bending was observed in response to CAF, although not statistically significant (Fig. 2A), and this indicates an increase in turns to avoid an aversive cue. Upon exposure to denatonium, mouth-hook contractions did not show a significant difference from the control (Fig. 2A, left), while bending increased (Fig. 2A, middle). These behavioral responses were consistent with the behavior tests that showed a neutral response to denatonium in the ingestion assay, and an aversive response in the choice preference test. The frequency of body wall contractions was not affected on exposure to $\mathrm{CAF}$ or denatonium (Fig. $2 A$, right), indicating that larval locomotion remained unaffected. However, all indices were observed to decrease on exposure to nicotine, including a strong drop in measured locomotion (Fig. 2A). This indicates that nicotine has a toxic or a muscular relaxant effect on larvae, causing an overall inhibition of movement. Thus, these results show that the reason nicotine initially appeared to act as an attractant in choice preference assays (Fig. 1C) was due to larvae simply stopping movement after reaching the nicotine-containing quadrants.

In the adult fly, Gr33a was identified as an important coreceptor in bitter-sensitive neurons that act in sensing most non-volatile bitter chemicals, and the Gr33a mutant $\left(G r 33 a^{1}\right)$ fly is insensitive to most bitter chemicals (Moon et al., 2009). Gr33a mutant larvae did not show the changes in mouth-hook contraction and/or bending that were observed in control larvae toward denatonium or CAF (Fig. 2B), indicating that these bitter-driven behaviors are mediated by Gr33a-expressing gustatory receptor neurons (GRNs). Body wall contractions of Gr33a ${ }^{1}$ larvae were observed to drop in the presence of nicotine, similarly to wCS larvae (Fig. 2B), showing that nicotine indeed 

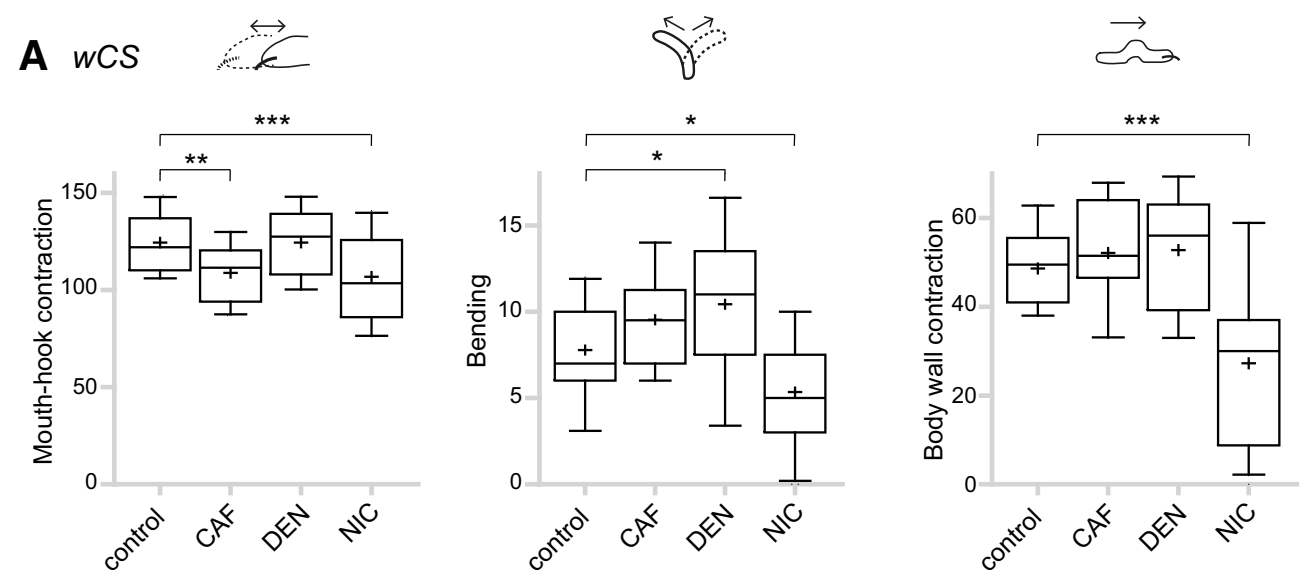

B $G r 33 a^{1}$
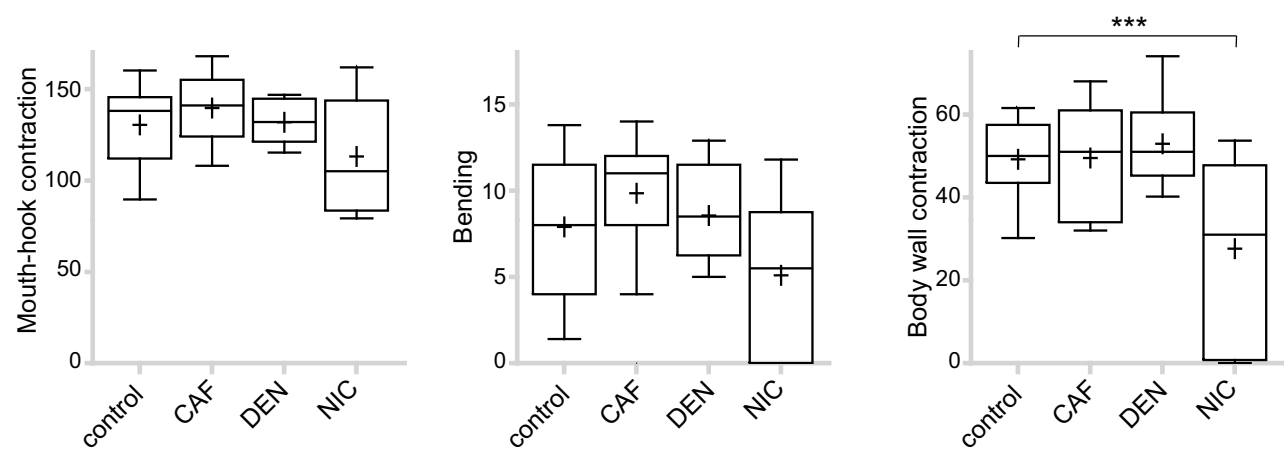

\section{C wCS}
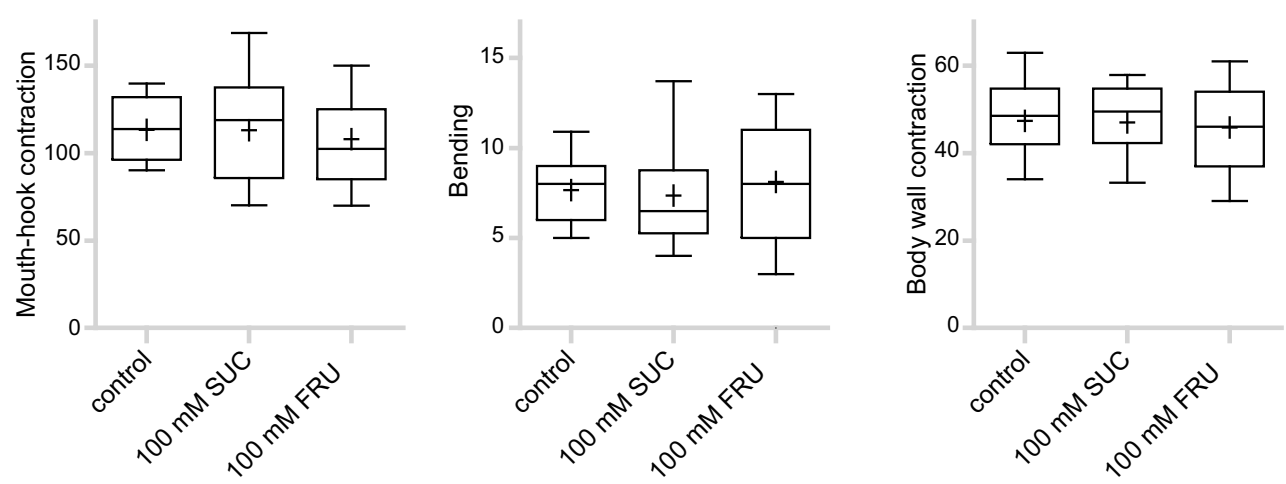

Figure 2. Measurement of larval behavioral responses to given tastants: mouth-hook contractions (left), bending (middle), and body wall contractions (right). Behavioral responses during $1 \mathrm{~min}$ for larvae placed on agarose plates containing the indicated tastants, compared with larvae placed on agarose-only control plates. $\boldsymbol{A}$, Results for wCS larvae. For each data point, $16<n<40 ; * p<0.05$, ** $p<0.01$, **** $<0.001$ versus control, one-way ANOVA followed by Dunnett's multiple comparison test. B, Results for Gr33a ${ }^{1}$ larvae. For each data point, $16<n<40 ; * * * p<0.001$ versus control, one-way ANOVA followed by Dunnett's multiple comparison test. $\boldsymbol{C}$, Behavioral responses of wCS larvae to the indicated sugars and control. For each data point, $16<n<40$.

inhibits larval movement. This also leads to a subsequent tendency of decrease in mouth-hook contractions and bending (Fig. 2B). In contrast to bitter chemicals, the measured phenotypes did not change noticeably on exposure to sugar (Fig. 2C), indicating that these behaviors are not useful parameters to measure feeding enhancement to attractive cues, likely because larvae feed by default.

\section{DP1 neurons are necessary and sufficient for CAF- induced ingestion reduction and avoidance}

Although both CAF and denatonium induce aversive behavior, CAF decreases both preference and ingestion, while denatonium has a strong inhibitory effect on only preference. This suggests that independent circuits may exist to detect different bitter tastants and to mediate distinct behavioral steps in feeding. To examine this 

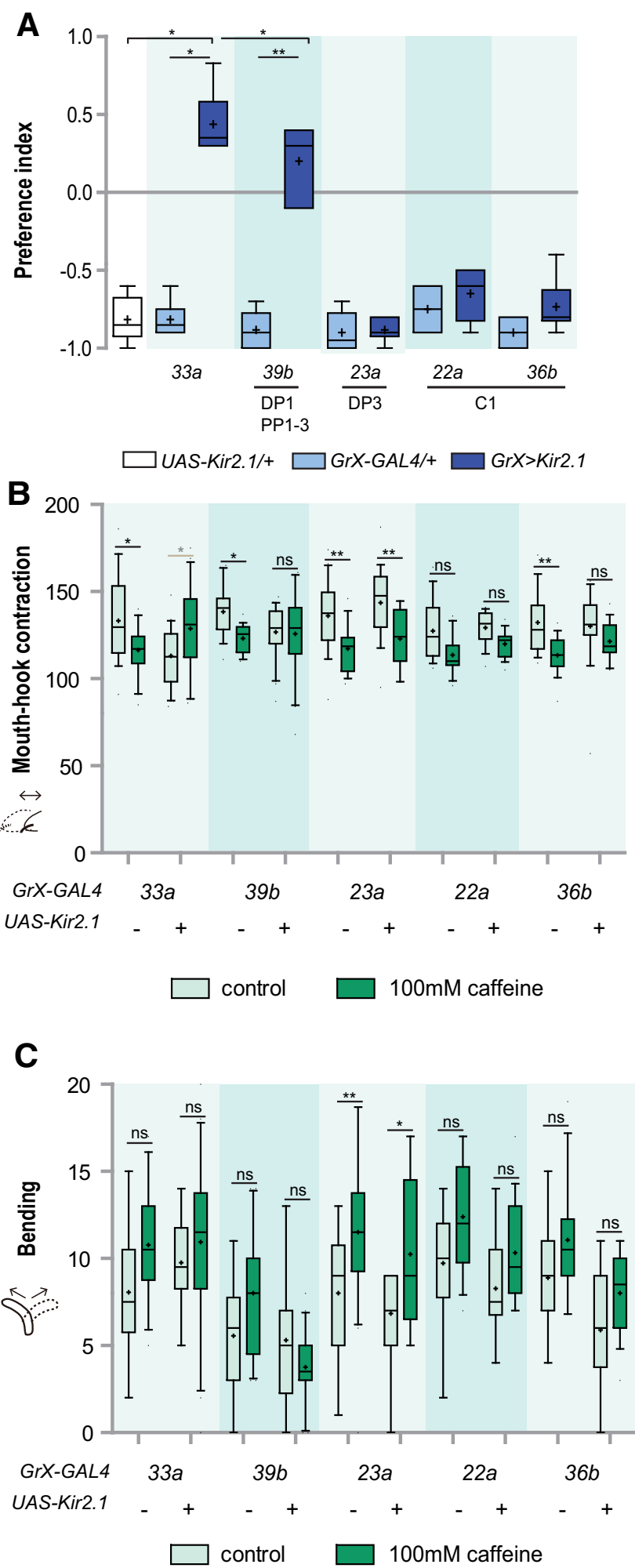

Figure 3. A subset of pharyngeal neurons including DP1 mediates CAF-induced avoidance and changes in mouth-hook contraction and bending behaviors. $\boldsymbol{A}$, Comparison of preference behavior in response to $100 \mathrm{~mm}$ CAF when Gr-GAL4 drivers specifically expressed in the GRNs listed under the underlines were used to block neuronal activity. For each data point, $n=6$. $* p<0.05, * * p<0.01$, Kruskal-Wallis test followed by Dunn's multiple comparison test. $\boldsymbol{B}$, Comparison of reduction of possibility and identify the initial sensory neurons of the neural circuits that mediate choice preference or ingestion, we aimed to identify differences in the cells that detect $\mathrm{CAF}$ and denatonium. For this purpose, we first used specific Gr-GAL4 drivers and the inward-rectifier potassium channel UAS-Kir2.1 to inhibit the activity of specific larval gustatory neurons.

In a previous study, we found that a specific pair of neurons in the DPS called DP1 is necessary and sufficient for CAF-mediated decrease in ingestion (Choi et al., 2016). An independent study found that the same neurons mediate CAF avoidance (Apostolopoulou et al., 2016). The DP1 neuron expresses the Gr33a-, Gr39b-, and Gr22dGAL4 drivers (Choi et al., 2016). Consistent with our previous study, CAF induces avoidance in the present choice preference assay, and this aversive effect is reversed when the activity of Gr33a- or Gr39b-GAL4-expressing neurons is inhibited, but not when inhibiting DP3 or C1 neurons (Fig. $3 A$ ). Also, CAF-induced decrease of mouthhook contractions was reversed on inhibition of Gr33a- or Gr39b-GAL4-expressing neuron activity (Fig. 3B). In contrast, CAF-induced decrease of mouth-hook contractions was unaffected on inhibition of the DP3 neuron (Fig. 3B). A similar trend was observed on inhibition of the $\mathrm{C} 1$ neuron, albeit the changes were not statistically significant (Fig. 3B). Although the variation in CAF-induced increase in bending was generally too large to come to a statistically significant conclusion, a general tendency of reversal was observed on inhibition of Gr39b-GAL4-expressing neuron (DP1) activity, but not on inhibition of DP3 or C1 neurons (Fig. $3 C$ ). Thus, DP1 is the main mediator of CAFinduced avoidance and changes in ingestion, mouthhook contraction, and bending behaviors.

Next, we attempted to rescue behavioral defects in Gr33a larvae that do not show CAF-sensitive behaviors, by expressing a wild-type Gr33a transgene in specific GRNs. Gr33a ${ }^{1}$ behavior in choice preference was rescued on expression of Gr33a in Gr33a-, Gr39b-, or Gr22dGAL4-expressing neurons (Fig. 4A). DP1 is commonly expressed by all three of these Gr-GAL4 drivers. In the case of mouth-hook contractions, Gr33a ${ }^{1}$ defects were only rescued when Gr33a was expressed in Gr33a-GAL4-expressing neurons, although when Gr33a was expressed in only DP1, mouth-hook contractions tended to decrease

\section{continued}

mouth-hook contractions in response to $100 \mathrm{~mm}$ CAF when $\mathrm{Gr}$ GAL4 drivers specifically expressed in the TO or pharyngeal organs were used to block GRN activity. For each data point, $17<n<30 ; * p<0.05$, $* * p<0.01$, two-way ANOVA followed by the Bonferroni post hoc test. Gr33a>Kir2.1 larvae in response to $100 \mathrm{~mm}$ CAF was marked in a gray asterisk to distinguish it from other data, since mouth-hook contractions were increased compared with other data. ns, not significant. $\boldsymbol{C}$, Comparison of increase of bending in response to $100 \mathrm{~mm}$ CAF when Gr-GAL4 drivers specifically expressed in the TO or pharyngeal organs were used to block GRN activity. For each data point, $17<n<30 ; * p<0.05$, $* * p<0.01$ versus control, twoway ANOVA followed by the Bonferroni post hoc test. ns, not significant. + and - indicate whether the transgenes are present or absent. 
A $G r 33 a^{1}$

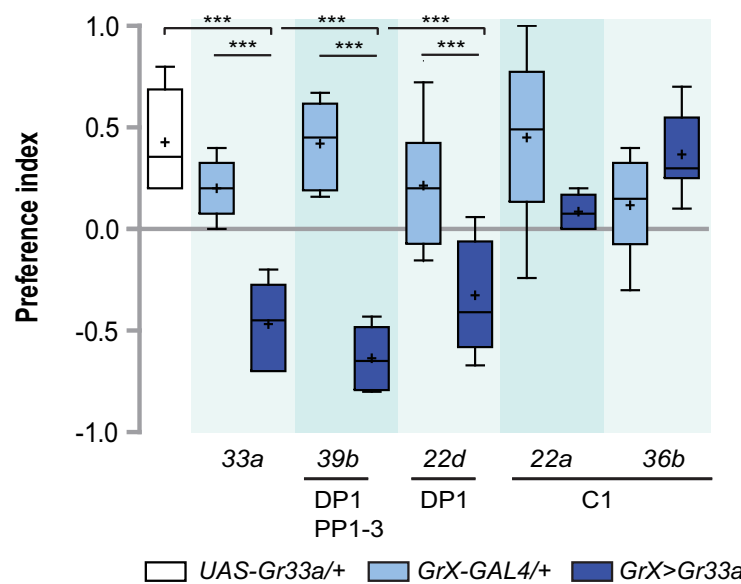

B $\mathrm{Gr}^{3} 3 a^{1}$

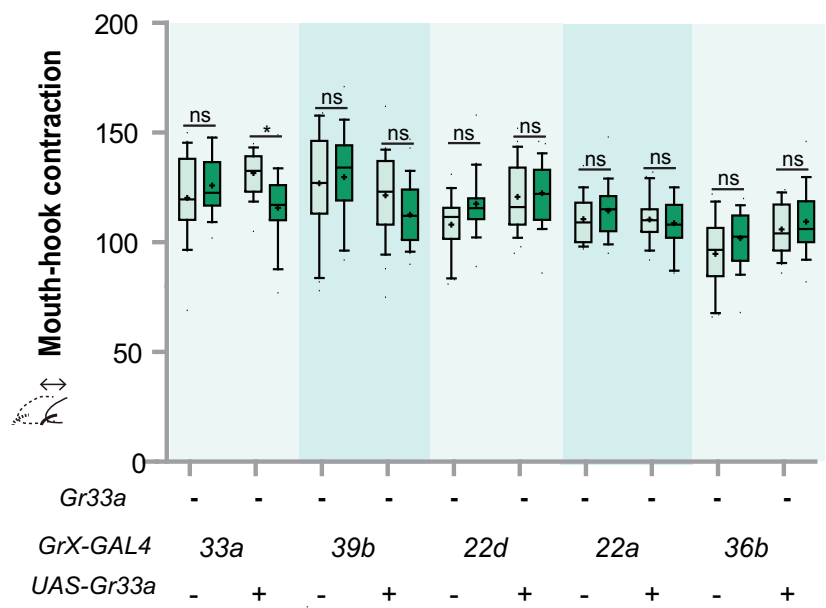

$\square$ control $\square$ 100mM caffeine

C Gr33a $^{1}$

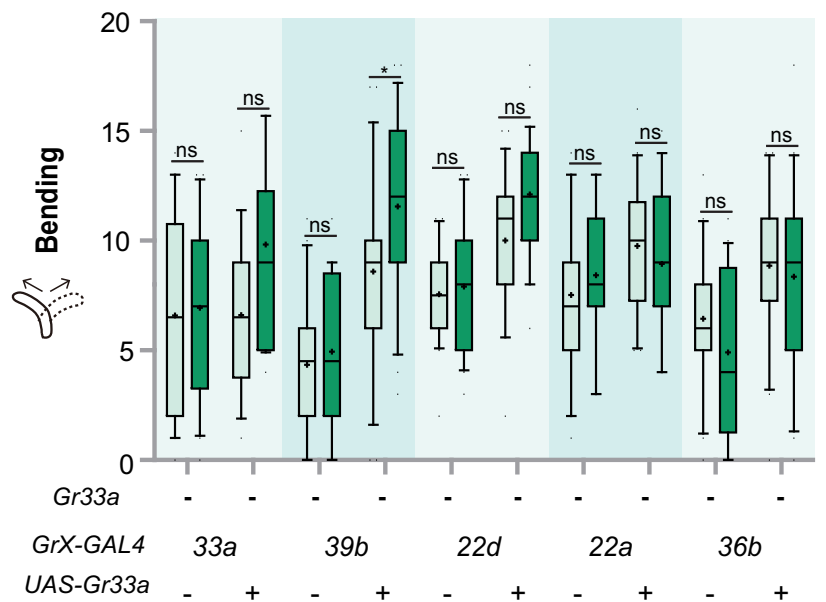

control $\square$ 100mM caffeine

Figure 4. DP1 is sufficient for CAF-induced aversive responses in ingestion and choice preference. $\boldsymbol{A}$, Choice preference in response to $100 \mathrm{~mm}$ CAF on expression of Gr33a in the Gr33a mutant using the indicated Gr-GAL4 drivers for specific on exposure to CAF compared with the GAL4-only control (Fig. 4B). Also, in the case of bending, Gr33a ${ }^{1}$ defects were only rescued when Gr33a was expressed in Gr39b-GAL4-expressing neurons, while bending showed a tendency to increase when Gr33a was expressed in DP1 and not when Gr33a was expressed in the $C 1$ neurons (Fig. 4C). Thus, Gr33a function in DP1 neurons is sufficient for all types of CAF-driven larval aversive behavior tested.

\section{Gustatory neurons in the TO mediate denatonium- induced avoidance behavior}

Our behavioral experiments indicated that denatonium did not affect ingestion and only affected choice preference. The Drosophila larval C7 neuron is known to be necessary and sufficient for denatonium sensing (van Giesen et al., 2016b). Also, Gr59c is known to function in denatonium sensing in the adult fly labellum (Weiss et al., 2011; Freeman et al., 2014; Sung et al., 2017), and Gr59c-GAL4 is specifically expressed in only one pair of larval GRNs, the $\mathrm{C} 1$ neuron pair, which houses expression of the highest number of Gr-GAL4 drivers in the TO, the major external gustatory organ of Drosophila larva (Kwon et al., 2011). Therefore, we tested the roles of the TO $C 1$ and $C 7$ neurons in denatonium sensing.

We used 3 Gr-GAL4 drivers (Gr22a-, Gr28b.e-, and Gr59c-GAL4) that are only expressed in C1 and C7-GAL4 to inhibit the activity of either $\mathrm{C} 1$ or $\mathrm{C} 7$, or both $\mathrm{C} 1$ and C7 simultaneously, and examined larval preference to denatonium. Avoidance of denatonium decreased on inhibition of either $\mathrm{C} 1$ or $\mathrm{C} 7$, and an additive decrease was observed on inhibition of both $\mathrm{C} 1$ and $\mathrm{C} 7$ (Fig. 5A). Consistent with our observations that denatonium did not affect mouth-hook contractions (Fig. 2), inhibition of $\mathrm{C} 1$ and $\mathrm{C} 7$ during denatonium exposure did not affect the mouth-hook contractions of larvae (Fig. 5B). In contrast, inhibition of either $\mathrm{C} 1$ or $\mathrm{C} 7$ resulted in a tendency to decrease denatonium-induced increase of larval bending behavior (Fig. 5C). These results suggest that $\mathrm{C} 1$ and $\mathrm{C} 7$ may work together to sense denatonium and induce avoidance behavior in wCS larvae. Consistently, intracellular calcium concentration was increased in these neurons during stimulation with denatonium (Fig. $5 D-F)$.

\section{continued}

expression in the GRNs listed under the underlines. For each data point, $6<n<12$; *** $<<0.001$ versus GAL4 and UAS control, one-way ANOVA followed by the Newman-Keuls method. $\boldsymbol{B}$, Comparison of reduction of mouth-hook contractions in response to $100 \mathrm{~mm}$ CAF on expression of Gr33a in the Gr33a mutant using the indicated Gr-GAL4 drivers for specific expression in the GRNs. For each data point, $18<n<28$; $* p<0.05$, two-way ANOVA followed by the Bonferroni post hoc test. ns, not significant. $\boldsymbol{C}$, Comparison of increase in bending in response to $100 \mathrm{~mm}$ CAF on expression of $\mathrm{Gr} 33 \mathrm{a}$ in the Gr33a mutant using the indicated Gr-GAL4 drivers for specific expression in the GRNs. For each data point, $18<n<28 ; * p<0.05$, two-way ANOVA followed by the Bonferroni post hoc test. ns, not significant. + and - indicate whether transgenes are present or absent. 
A

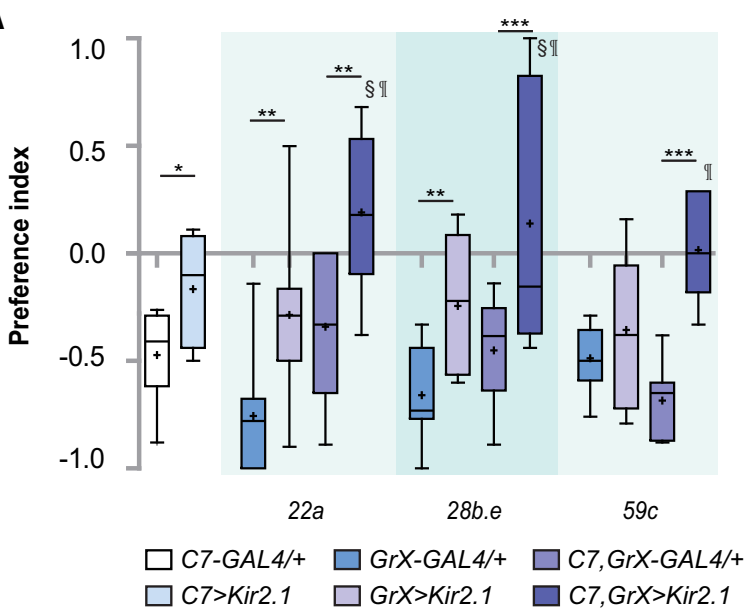

B

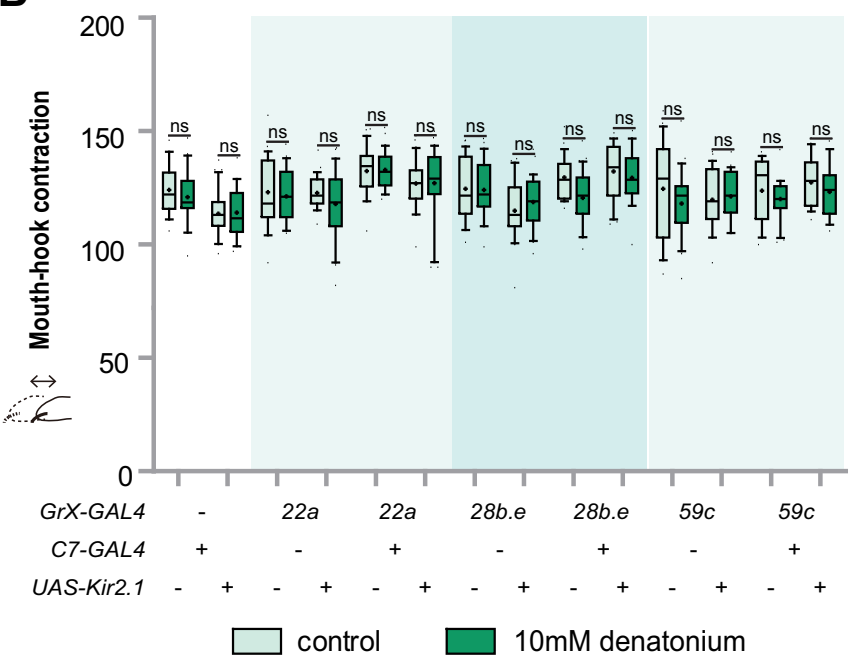

C

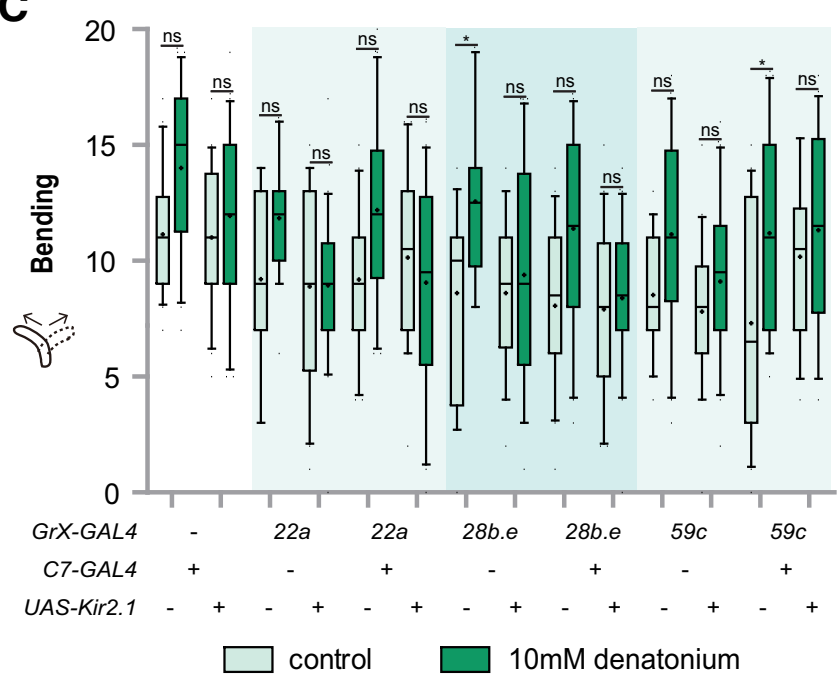

D
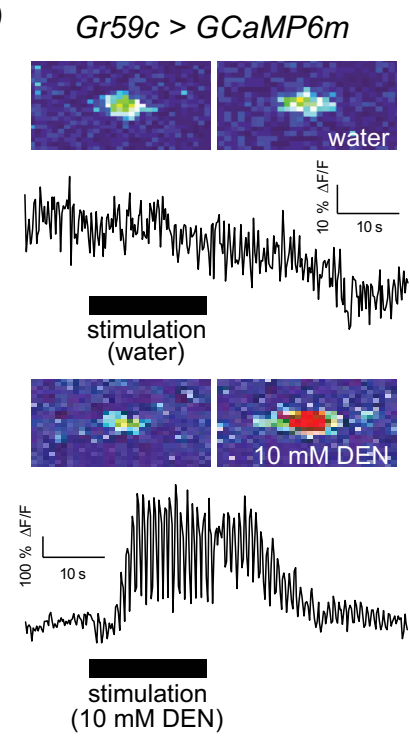

E C7 > GCaMP6m
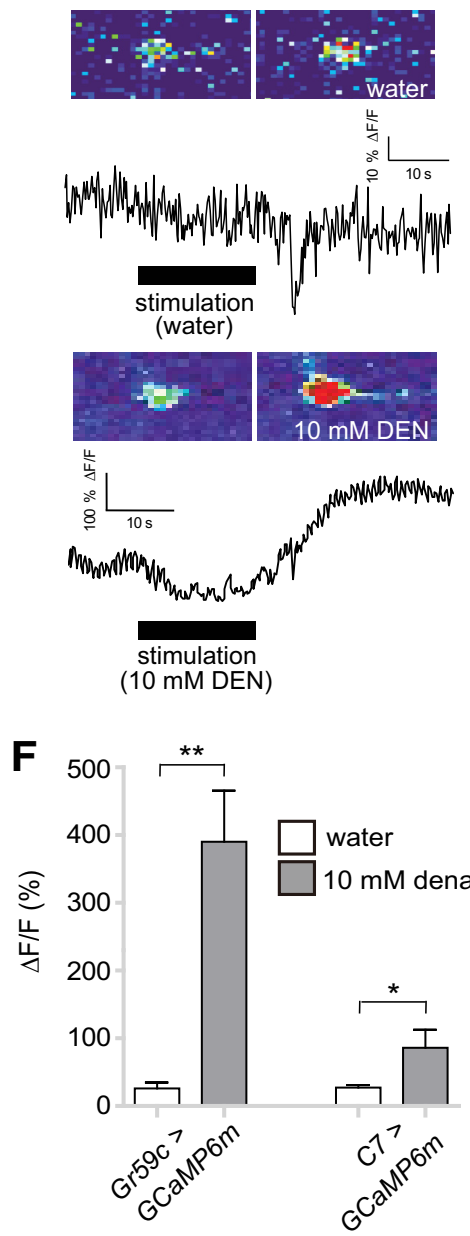

Figure 5. Two pairs of GRNs in the TO, C1 and C7, detect denatonium to induce avoidance. $\boldsymbol{A}$, Comparison of preference behavior in response to $10 \mathrm{~mm}$ denatonium when GAL4 drivers specifically expressed in C1 or C7, GRNs in the TO, were used to block neuronal activity. For each data point, $6<n<10 ; * p<0.05, * * p<0.01, * * * p<0.001$, one-way ANOVA followed by uncorrected Fisher's LSD test. Symbols $\S$ and $\uparrow$ each represent the significance versus C7>Kir2.1 and GrX $>$ Kir2.1. B, Comparison of reduction of mouth-hook contractions in response to $10 \mathrm{~mm}$ denatonium when GAL4 drivers specifically expressed in the TO were used to 
continued

block GRN activity in $\mathrm{C} 1$ and/or C7. For each data point, $18<n<20$. Two-way ANOVA followed by the Bonferroni post hoc test. ns, not significant. $\boldsymbol{C}$, Comparison of increase of bending in response to $10 \mathrm{~mm}$ denatonium when GAL4 drivers specifically expressed in the TO were used to block GRN activity in C1 and/or C7. For each data point, $18<n<20$; $* p<0.05$, two-way ANOVA followed by the Bonferroni post hoc test. ns, not significant. "+" and "-" indicate whether transgenes are present or absent. $\boldsymbol{D}$, $\boldsymbol{E}$, Calcium currents can be measured in the $C 1(\boldsymbol{D})$ and $C 7(\boldsymbol{E})$ neurons before and during the application of $10 \mathrm{~mm}$ denatonium using the genetically encoded calcium sensor GCaMP6m. $\boldsymbol{F}$, TO C1 and C7 neurons, labeled by Gr59c-GAL4 and C7-GAL4, respectively, showed neuronal activity to $10 \mathrm{~mm}$ denatonium. For each data point, $6<n<30 ; * p<0.05$, $* * p<0.01$, Mann-Whitney $U$ test pairwise comparisons of water control and $10 \mathrm{~mm}$ denatonium.

\section{Activation of DP1 is sufficient to cause the suppression of ingestion}

We tested whether activation of the DP1 neuron by denatonium could induce a change in ingestion, which is not normally affected by denatonium. For this purpose, we used Gr59c, which is known to be critical for denatonium sensing in the adult fly (Weiss et al., 2011; Freeman et al., 2014; Sung et al., 2017). Based on GAL4 reporter expression, Gr59c is not expressed in larval pharyngeal neurons (Kwon et al., 2011). We expressed Gr59c in DP1 using two Gr-GAL4 drivers, Gr22d-and Gr39b-GAL4, and examined the ingestion of denatonium. Larvae were observed to show a decrease in ingestion compared with the control (Fig. $6 A$ ), as well as a decrease in mouth-hook contractions (Fig. 6B). In addition, DP1 neurons ectopically expressing Gr59c were observed to show an increase in cellular calcium levels on exposure to denatonium (Fig. $6 C-E$ ). These results indicate that activation of the pharyngeal GRN DP1 by a specific substrate is sufficient to suppress the ingestion of that substrate. Thus, wCS larvae exposed to denatonium show a change only in choice preference but not ingestion, because denatonium is only detected by the TO and not by the pharyngeal organs.

\section{Discussion}

A general assumption would be that a tastant would cause a similar response in ingestion and choice preference behavior, in either a positive or negative manner. However, our findings corroborate that certain tastants elicit divergent ingestion and choice preference behavior. Combining molecular genetic tools, behavioral assays, and genetically encoded calcium sensors to assess neuronal activity, our results provide evidence that relatively independent neural systems appear to regulate the two initial processes of feeding in Drosophila larva: searching for palatable food, i.e., choice preference, and eating the selected food, i.e., ingestion. We show that a subset of gustatory neurons housed in the TO, the external gustatory organ of Drosophila larva, detect denatonium and induce avoidance behavior, and DP1, a specific pair of GRNs in the dorsal pharyngeal organ, plays a major role in regulating both ingestion and avoidance in response to CAF.

The TO of Drosophila larva is located at the tip of the cephalic lobes, and is thus anatomically likely to be the first organ to contact external stimuli and subsequently cause a change in movement to regulate the initial step of feeding. Similarly, pharyngeal sensilla are located between the external sense organs and digestive organs, and are thus anatomically likely to act in maintaining the ingestion of appetitive foods while stopping ingestion and causing avoidance of aversive cues such as bitter toxins. It could be advantageous for ingestion to be predominantly controlled by pharyngeal sense organs, rather than by external organs, since animals can try out a potential food source before making their decision, rather than blindly avoiding it. This could be a particularly advantageous strategy for insect larvae whose main purpose is to feed. Also, the difference in behavioral responses elicited by the $\mathrm{C} 1$ and $\mathrm{C} 7$ neurons in the TO and DP1 in the pharyngeal sense organs is likely linked to the difference in brain projection patterns of GRNs from the TO and pharyngeal GRNs from the larval SEZ (Kwon et al., 2011), with the distinct projection areas of the brain taste center likely being linked to different circuits, resulting in distinct behavioral outputs.

In Drosophila larvae, choice and ingestion have generally been grouped together and studied as a group of reflexive behaviors (Schipanski et al., 2008; El-Keredy et al., 2012; Rohwedder et al., 2012; Apostolopoulou et al., 2014). Sugar processing provides another intriguing example of divergence between choice and ingestion. Larvae generally show increased preference and feeding when exposed to increasing concentrations of fructose or sucrose. At extremely high concentrations such as $2 \mathrm{M}$ or $4 \mathrm{M}$, larvae still exhibit preference in terms of choice, but show suppression of feeding (or ingestion, as we denote it here; Schipanski et al., 2008; Rohwedder et al., 2012). Since this suppression of ingestion could be due to high viscosity and/or osmolarity, a direct comparison to the processing of aversive tastants such as bitter chemicals is difficult. However, this example nonetheless provides evidence that relatively independent circuits exist to determine choice and ingestion. Using bitter tastants, we find that choice and ingestion can manifest in clearly divergent behaviors to the same compounds and elucidate the cellular basis of these observations. Similarities to the observation that external sense organs and pharyngeal organs appear to be involved in somewhat independent behavioral output can be seen in sugar consumption in the adult fly. The activation of sweet GRNs in the legs and labellum initiates feeding behaviors including the proboscis extension reflex, and pharyngeal sweet GRNs play an important role in directing the sustained consumption of sweet compounds (LeDue et al., 2015).

Most of the 22 putative bitter tastants tested here, including CAF, cause negative effects in choice preference and ingestion. Nicotine caused a positive P.I. in the choice preference assay. Although we cannot completely rule out the possibility that nicotine could act as an attractive 
A

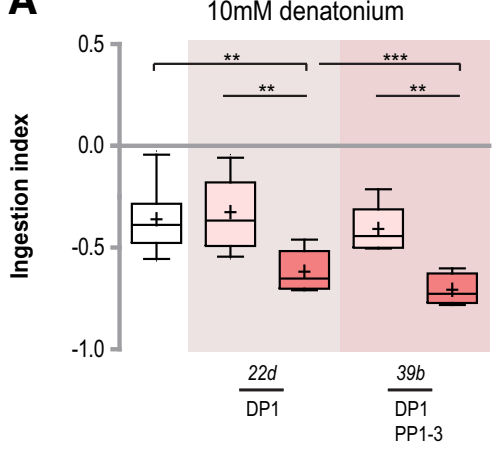

B

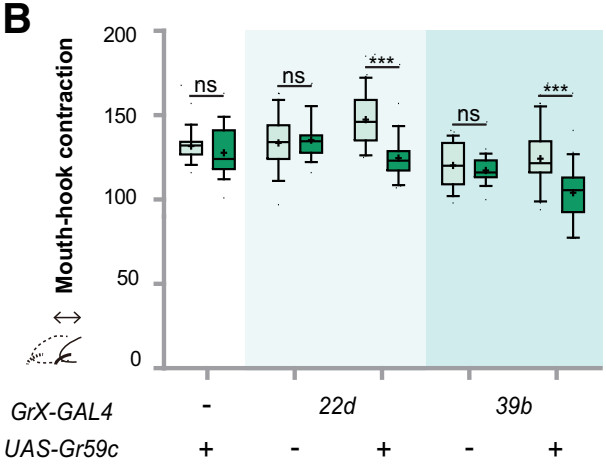

$\square$ control $\square$ 10mM denatonium

$\square$ UAS-Gr59c/+ $\square$ GrX-GAL4/+ $\square$ GrX>Gr59c

C

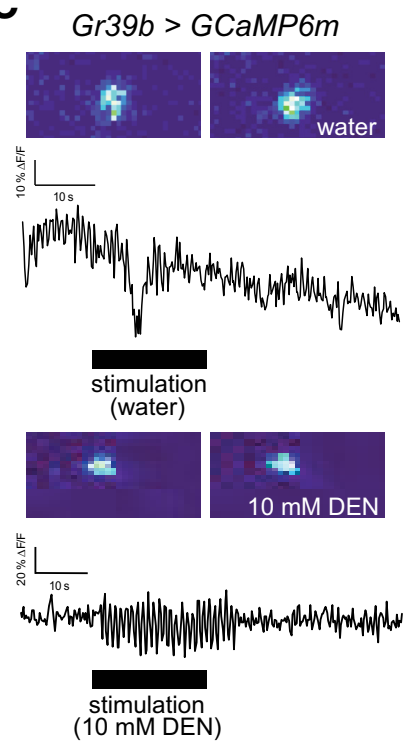

D

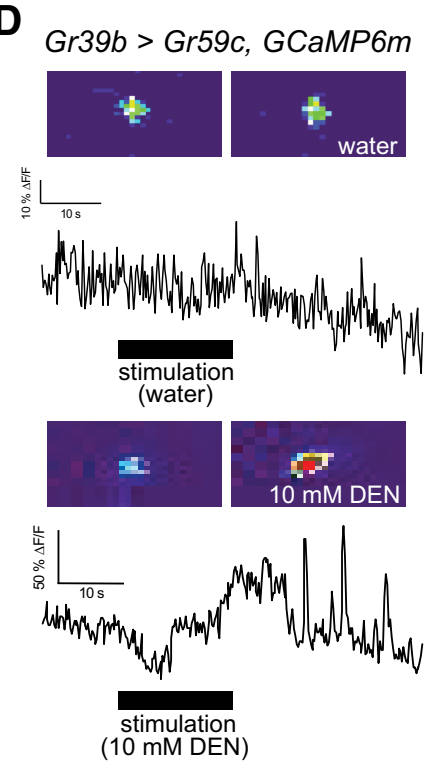

E

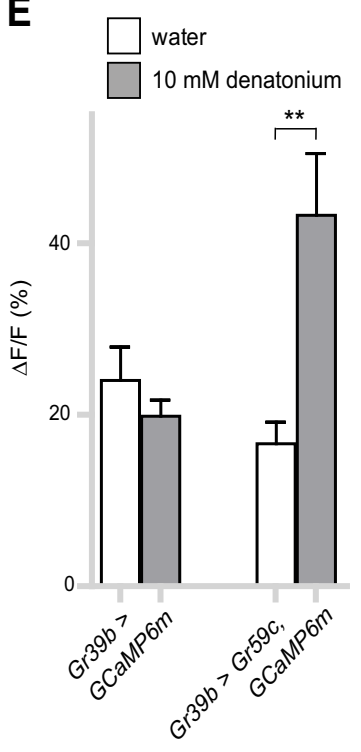

Figure 6. Detection of denatonium by DP1, a pair of dorsal pharyngeal neurons, causes suppression of ingestion. $\boldsymbol{A}$, Comparison of ingestion in response to $10 \mathrm{~mm}$ denatonium when Gr22d-GAL4 and Gr39b-GAL4 drivers were used to ectopically express Gr59c in DP1. For each data point, $6<n<14 ; * * p<0.01$, ***p $<0.001$ versus GAL4 and UAS control, one-way ANOVA followed by the Newman-Keuls method. B, Comparison of reduction of mouth-hook contractions in response to 10 mm denatonium when Gr22dGAL4 and Gr39b-GAL4 drivers were used to ectopically express Gr59c in DP1. For each data point, $18<n<20 ; * * * p<0.001$, twoway ANOVA followed by the Bonferroni post hoc test. ns, not significant. + and - indicate whether transgenes are present or absent. $\boldsymbol{C}, \boldsymbol{D}$, Calcium currents were measured in DP1 neurons with Gr39b-GAL4 used to express GCaMP6m only (C) or GCaMP6m and $\mathrm{Gr} 59 \mathrm{c}$ together $(\boldsymbol{D})$ before and during the application of $10 \mathrm{~mm}$ denatonium. $\boldsymbol{E}$, Changes in neuronal activity in response to 10 $\mathrm{mm}$ denatonium was measured in the DP1 neuron with ectopically expressed Gr59c. For each data point, $6<n<11 ; * * p<0.01$, Mann-Whitney $U$ test pair-wise comparisons of water control and $10 \mathrm{~mm}$ denatonium.

chemosensory cue at low concentrations, we found that nicotine inhibits the movement of larvae in our experimental setup. Larvae strongly avoid denatonium, but once they sample denatonium-containing food, they ingest it. This ingestion likely occurs because denatonium is added to the agarose of the entire plate, whereas larvae probably would not ingest as much if they had the choice. Nevertheless, our results suggest that this larval response to denatonium is due to the existence of a functional receptor complex for denatonium in the TO, which does not exist in the pharyngeal sense organs, or at the very least the DP1 neuron. Consistently, ectopic expression of GR59c in DP1 caused a novel calcium response to denatonium and suppression of ingestion in response to denatonium. Some remaining questions regarding sensing of denatonium merit further study. Avoidance to denatonium is defective when either $\mathrm{C} 1$ or $\mathrm{C} 7$ is inactivated, indicating that $\mathrm{C} 1$ and $\mathrm{C} 7$ are not redundant in terms of behavior. It is possible that a certain threshold of neuronal activity is required to elicit behavior, or inactivation of one neuron may cause a change in the functions of other GRNs. Although a numerically simple system, larval GRNs also have a multimodal character (van Giesen et al., 2016b), and as such a more complicated mechanism might be involved. Also, in the bitter sensing neurons of the adult labellum, two complexes, GR32a/GR66a/GR59c and GR32a/GR66a/GR22e, are each sufficient to confer a response to denatonium (Sung et al., 2017). Based on 
Gr-GAL4 expression, the larval DP1 neuron expresses Gr22e, but not Gr59c (Choi et al., 2016), but is not capable of detecting denatonium. This suggests that the GRNs of the larva and adult fly possess different cellular contexts, which could be interesting to unravel. An interesting remaining question is if Gr59c is solely responsible for denatonium sensing in the larval $\mathrm{C} 1$ neuron or if the existing Gr22e can rescue denatonium sensing in Gr59c mutants. This would indicate that Gr22e needs a specific co-receptor repertoire for denatonium detection and could help elucidate coding differences in the larva versus the adult fly.

The levels at which distinct bitter compounds are detected might reflect the ecological niche of the animal and the toxicity level of a given tastant. Our results suggest that information from the DP1 neuron is processed in a circuit that results in negative and aversive behavior in ingestion and choice preference to CAF. The C1 and C7 neuron in the TO elicit avoidance to denatonium in choice preference behavior. Thus, these results suggest that distinct sensory neurons appear to have distinct sensory roles, likely through the expression of specific receptors or specific groups of receptors. Sensory information detected by these sensory neurons appears to be processed through distinct circuits in the central nervous system to mediate changes in ingestion or choice behavior. It is yet unclear whether the different circuits interact to result in a final behavioral output. Further examination of the potential connections between the external and pharyngeal gustatory neurons and interneurons or motor neurons in the brain may provide insight into the overall neural circuit that regulates feeding and locomotion.

\section{References}

Apostolopoulou AA, Mazija L, Wüst A, Thum AS (2014) The neuronal and molecular basis of quinine-dependent bitter taste signaling in Drosophila larvae. Front Behav Neurosci 8:6.

Apostolopoulou AA, Rist A, Thum AS (2015) Taste processing in Drosophila larvae. Front Integr Neurosci 9:50.

Apostolopoulou AA, Köhn S, Stehle B, Lutz M, Wüst A, Mazija L, Rist A, Galizia CG, Lüdke A, Thum AS (2016) Caffeine taste signaling in Drosophila larvae. Front Cell Neurosci 10:193.

Baines RA, Uhler JP, Thompson A, Sweeney ST, Bate M (2001) Altered electrical properties in Drosophila neurons developing without synaptic transmission. J Neurosci 21:1523-1531.

Bhatt PK, Neckameyer WS (2013) Functional analysis of the larval feeding circuit in Drosophila. J Vis Exp (81):e51062.

Chen TW, Wardill TJ, Sun Y, Pulver SR, Renninger SL, Baohan A, Schreiter ER, Kerr RA, Orger MB, Jayaraman V, Looger LL, Svoboda K, Kim DS (2013) Ultrasensitive fluorescent proteins for imaging neuronal activity. Nature 499:295-300.

Choi J, van Giesen L, Choi MS, Kang K, Sprecher SG, Kwon JY (2016) A pair of pharyngeal gustatory receptor neurons regulates caffeine-dependent ingestion in Drosophila larvae. Front Cell Neurosci 10:181

Colomb J, Grillenzoni N, Ramaekers A, Stocker RF (2007) Architecture of the primary taste center of Drosophila melanogaster larvae. J Comp Neurol 502:834-847.

El-Keredy A, Schleyer M, König C, Ekim A, Gerber B (2012) Behavioural analyses of quinine processing in choice, feeding and learning of larval Drosophila. PLoS One 7:e40525.

Freeman EG, Wisotsky Z, Dahanukar A (2014) Detection of sweet tastants by a conserved group of insect gustatory receptors. Proc Natl Acad Sci USA 111:1598-1603.
Gendre N, Lüer K, Friche S, Grillenzoni N, Ramaekers A, Technau GM, Stocker RF (2004) Integration of complex larval chemosensory organs into the adult nervous system of Drosophila. Development 131:83-92.

Gerber B, Stocker RF (2007) The Drosophila larva as a model for studying chemosensation and chemosensory learning: a review. Chem Senses 32:65-89.

Hernandez-Nunez L, Belina J, Klein M, Si G, Claus L, Carlson JR, Samuel AD (2015) Reverse-correlation analysis of navigation dynamics in Drosophila larva using optogenetics. Elife 4:e06225.

Jaeger AH, Stanley M, Weiss ZF, Musso PY, Chan RC, Zhang H, Feldman-Kiss D, Gordon MD (2018) A complex peripheral code for salt taste in Drosophila. Elife 7:e37167.

Joshi A, Mueller LD (1988) Evolution of higher feeding rate in Drosophila due to density-dependent natural selection. Evolution 42:1090-1093.

Kim H, Choi MS, Kang K, Kwon JY (2016) Behavioral analysis of bitter taste perception in Drosophila larvae. Chem Senses 41:85-94.

König C, Schleyer M, Leibiger J, El-Keredy A, Gerber B (2014) Bittersweet processing in larval Drosophila. Chem Senses 39:489-505.

Kwon JY, Dahanukar A, Weiss LA, Carlson JR (2011) Molecular and cellular organization of the taste system in the Drosophila larva. J Neurosci 31:15300-15309.

LeDue EE, Chen YC, Jung AY, Dahanukar A, Gordon MD (2015) Pharyngeal sense organs drive robust sugar consumption in Drosophila. Nat Commun 6:6667.

Liu L, Leonard AS, Motto DG, Feller MA, Price MP, Johnson WA, Welsh MJ (2003) Contribution of Drosophila DEG/ENaC genes to salt taste. Neuron 39:133-146.

Mishra D, Miyamoto T, Rezenom YH, Broussard A, Yavuz A, Slone J, Russell DH, Amrein H (2013) The molecular basis of sugar sensing in Drosophila larvae. Curr Biol 23:1466-1471.

Moon SJ, Lee Y, Jiao Y, Montell C (2009) A Drosophila gustatory receptor essential for aversive taste and inhibiting male-to-male courtship. Curr Biol 19:1623-1627.

Okusawa S, Kohsaka H, Nose A (2014) Serotonin and downstream leucokinin neurons modulate larval turning behavior in Drosophila. J Neurosci 34:2544-2558.

Pool AH, Scott K (2014) Feeding regulation in Drosophila. Curr Opin Neurobiol 29:57-63.

Python F, Stocker RF (2002) Immunoreactivity against choline acetyltransferase, gamma-aminobutyric acid, histamine, octopamine, and serotonin in the larval chemosensory system of Drosophila melanogaster. J Comp Neurol 453:157-167.

Rist A, Thum AS (2017) A map of sensilla and neurons in the taste system of Drosophila larvae. J Comp Neurol 525:3865-3889.

Rohwedder A, Pfitzenmaier JE, Ramsperger N, Apostolopoulou AA, Widmann A, Thum AS (2012) Nutritional value-dependent and nutritional value-independent effects on Drosophila melanogaster larval behavior. Chem Senses 37:711-721.

Schipanski A, Yarali A, Niewalda T, Gerber B (2008) Behavioral analyses of sugar processing in choice, feeding, and learning in larval Drosophila. Chem Senses 33:563-573.

Shen P (2012) Analysis of feeding behavior of Drosophila larvae on solid food. Cold Spring Harb Protoc 2012:pdb.prot069328.

Singh RN, Singh K (1984) Fine structure of the sensory organs of Drosophila melanogaster meigen larva (Diptera: Drosophilidae). Int J Insect Morphol Embryol 13:255-273.

Stewart S, Koh TW, Ghosh AC, Carlson JR (2015) Candidate ionotropic taste receptors in the Drosophila larva. Proc Natl Acad Sci USA 112:4195-4201.

Stocker RF (1994) The organization of the chemosensory system in Drosophila melanogaster: a review. Cell Tissue Res 275:3-26.

Sung HY, Jeong YT, Lim JY, Kim H, Oh SM, Hwang SW, Kwon JY, Moon SJ (2017) Heterogeneity in the Drosophila gustatory receptor complexes that detect aversive compounds. Nat Commun 8:1484.

Thorne N, Amrein H (2008) Atypical expression of Drosophila gustatory receptor genes in sensory and central neurons. J Comp Neurol 506:548-568. 
van Giesen L, Neagu-Maier GL, Kwon JY, Sprecher SG (2016a) A microfluidics-based method for measuring neuronal activity in Drosophila chemosensory neurons. Nat Protoc 11:23892400.

van Giesen L, Hernandez-Nunez L, Delasoie-Baranek S, Colombo M, Renaud P, Bruggmann R, Benton R, Samuel AD, Sprecher SG (2016b) Multimodal stimulus coding by a gustatory sensory neuron in Drosophila larvae. Nat Commun $7: 10687$.
Weiss LA, Dahanukar A, Kwon JY, Banerjee D, Carlson JR (2011) The molecular and cellular basis of bitter taste in Drosophila. Neuron 69:258-272.

Wu Q, Wen T, Lee G, Park JH, Cai HN, Shen P (2003) Developmental control of foraging and social behavior by the Drosophila neuropeptide Y-like system. Neuron 39:147-161.

Wu Q, Zhang Y, Xu J, Shen P (2005) Regulation of hunger-driven behaviors by neural ribosomal S6 kinase in Drosophila. Proc Natl Acad Sci USA 102:13289-13294. 